19 Revue d'histoire du XIXe siècle

Société d'histoire de la révolution de 1848 et des

révolutions du XIXe siècle

$38 \mid 2009$

Savoirs occultés : du magnétisme à l'hypnose

\title{
Introduction
}

Nicole Edelman

URL : http://journals.openedition.org/rh19/3869

DOI : $10.4000 /$ rh 19.3869

ISSN : $1777-5329$

Éditeur

La Société de 1848

Édition imprimée

Date de publication : 1 juillet 2009

Pagination : 11-15

ISSN : 1265-1354

Référence électronique

Nicole Edelman, «Introduction », Revue d'histoire du XIXe siècle [En ligne], 38 | 2009, mis en ligne le 03 septembre 2009, consulté le 22 septembre 2020. URL : http://journals.openedition.org/rh19/3869 ; DOl : https://doi.org/10.4000/rh19.3869 


\section{NICOLE EDELMAN}

\section{Introduction}

Le magnétisme animal, et plus encore le somnambulisme magnétique, n'ont encore été que peu étudiés par les historiens. Les origines du romantisme et de l'hypnose donnèrent lieu aux premiers travaux qui conduisirent à plusieurs publications à la fin des années $1960^{1}$ dont certaines sur «l'inventeur» du magnétisme animal : le médecin Franz Anton Mesmer (1734-1815). Le psychiatre Henri Frédéric Ellenberger appartient ainsi aux pionniers de l'exploration de ces domaines puisqu'il fut un des premiers à mettre au jour la multiplicité des pratiques et des phénomènes magnétiques en Europe. Son livre The Discovery of the Unconscious. The History and Evolution of Dynamic Psychiatry, publié pour la première fois en $1970^{2}$, réédité et traduit depuis dans de multiples langues, demeure toujours une référence essentielle par sa richesse factuelle et son érudition. Sur l'ampleur des effets politiques et idéologiques du magnétisme animal à la fin du XVIII siècle, le livre de l'historien Robert Darnton, Mesmerism and the End of the Enlighment in France publié en $1968^{3}$ fut lui aussi novateur et reste encore unique par son questionnement politique. Robert Darnton y soutient une thèse, portée par le titre de l'ouvrage, qui demeure féconde parce que problématique : l'historien y relie étroitement la fin des Lumières à l'émergence de courants spiritualistes issus du mesmérisme au début du XIX siècle. Il estime que le mesmérisme participe ainsi à la résolution de la question à laquelle sont confrontés bien des penseurs des Lumières après la Révolution française et la période de la Terreur. Prenant pour exemples le physiocrate Dupont de Nemours d'une part et Condorcet de l'autre, il oppose les deux solutions que ces hommes font alors émerger, le recours à un spiritualisme, à l'élaboration d'une théo-

1. Voir la bibliographie (très limitative et ne citant que les premiers ouvrages publiés sur ce thème) en fin d'introduction.

2. Henri Frédéric Ellenberger, The Discovery of the Unconscious. The History and Evolution of Dynamic Pychiatry, New York, Basic Books, 1970, traduction française À la découverte de l'inconscient. Histoire de la psychiatrie dynamique, Villeurbanne, SIMEP, 1974, (nouvelle édition Paris, Fayard, 1994, sous le titre Histoire de la découverte de l'inconscient).

3. Robert Darnton, Mesmerism and the End of the Enlighment in France, Cambridge (Mass.), Harvard University Press, 1968, traduction française La Fin des Lumières, Le mesmérisme et la révolution, Paris, Perrin, 1984. 
dicée naturelle pour le premier ${ }^{4}$ et à la croyance au Progrès qui triomphera de la superstition pour le second. Et Robert Darnton conclut : «Après la Terreur, les mesméristes peuvent être révolutionnaires comme Bonneville ou conservateurs comme Bergasse, mais ils ne bâtiront pas leurs temples sur les fondations de la raison. " 5

Ce numéro thématique de la Revue d'histoire du XIXe siècle se propose donc de revisiter ces phénomènes longtemps oubliés et qui, dans leur temps d'émergence, furent souvent occultés en tant que savoirs (et même condamnés en France) ou moqués en tant que croyances, peut-être parce qu'ils ouvraient sur une vision et une connaissance de l'homme et du monde difficiles, voire impossibles, à penser dans ces décennies post-révolutionnaires de remise en ordre sociale et politique. Savoirs occultés : du magnétisme à l'hypnose a pour ambition de montrer combien le succès du magnétisme animal fut grand à la fin du XVIII siècle et de quelles manières il se déclina de la fin du XVIII siècle jusqu'aux premières décennies du XIX ${ }^{e}$ siècle, en se déployant dans des espaces très divers, ceux du savoir comme ceux de la croyance. Ce numéro s'attache cependant essentiellement aux savoirs médicaux que le magnétisme animal a irrigués et qui ont transformé très lentement, et de manière non linéaire, l'observation et les soins de ce que nous appelons aujourd'hui les maladies nerveuses et mentales, avec des différences sensibles selon les espaces culturels et géographiques.

Le médecin Franz Anton Mesmer est directement à l'origine de ces phénomènes puisqu'il est le découvreur du «magnétisme animal». Incontestablement le mieux connu, aucun article n'en traite ici exclusivement, mais tous s'y réferent. Dans les dernières décennies du XVIII siècle, ce médecin autrichien ${ }^{6}$ a élaboré une théorie globale de la santé et de la maladie; l'ensemble de cette théorie et sa mise en pratique furent souvent nommés «mesmérisme». Sachant que la mauvaise circulation du fluide est, pour Mesmer, à l'origine des maladies, le rôle du médecin (qui doit, lui-même, posséder ce fluide en suffisance), est de supprimer ces obstacles en magnétisant le corps. Mesmer pratique d'abord à Vienne où il possède une riche demeure qu'il doit à la fortune de son épouse. Puis à la suite d'un scandale lié aux soins donnés pour faire recouvrer la vue à une jeune pianiste aveugle, Marie-Therèse Paradis, fille d'un conseiller impérial, il doit quitter la capitale viennoise. Il se rend alors à Paris où il arrive en 1778 et s'installe assez vite dans l'hôtel Bullion. Son succès y prend une telle ampleur qu'il invente un baquet pour pouvoir magnétiser plusieurs malades en même temps, dans une mixité que

4. Voir Pierre-Samuel Dupont de Nemours, Philosophie de l'univers, Paris, imprimerie Du Pont, 1793.

5. R. Darnton, La Fin des Lumières..., ouv. cité, p. 148.

6. Mesmer est né à Iznang sur le domaine du prince-évêque du lac de Constance (Bodensee) qui est alors rattaché à l'empire autrichien. Mais il est demandé par la faculté de Berlin qui le voit alors volontiers comme prussien après la condamnation du magnétisme en 1784. Enfin Mesmer se retire à la fin de sa vie en Suisse, pays pour lequel il proposera une constitution... 
beaucoup jugent immorale. Très vite, le mesmérisme suscite ainsi l'hostilité d'une partie de la médecine académique et en 1784, une enquête est ordonnée par le roi Louis XVI. Réalisée à la fois par des médecins et des membres de l'Académie royale des sciences, elle conclut à l'inexistence du fluide. Le magnétisme animal demeure cependant pratiqué ${ }^{7}$, même en France, d'autant plus qu'en 1784, se fonde à Paris et dans plusieurs villes françaises la "Société d'Harmonie" dont les membres sont seuls habilités à mesmériser, possédant le «secret» de Mesmer qu'ils ont acheté. Amand Marc Jacques de Chastenet, marquis de Puységur, appartient à cette société et c'est en voulant soigner l'un de ses valets de ferme qu'il découvre par hasard cette même année 1784, ce qu'il nomme le «somnambulisme magnétique». La Révolution française stoppe tout débat sur ces thèmes et Mesmer, disqualifié par la condamnation des commissions royales, ne continue pas ses cures à Paris. Il se déplace alors beaucoup, se rend à Vienne en 1791 pour la mort de son épouse, revient à Paris plusieurs fois, est invité à Berlin puis s'installe en Suisse, de 1809 à 1812 à Frauenfeld, puis à Constance et enfin à Meersburg où il meurt le 5 mars 1815 sur les bords même du lac où il était né ${ }^{8}$.

Mesmer, le magnétisme animal et le somnambulisme magnétique ont ainsi laissé des marques profondes dans l'histoire sociale et culturelle des sciences médicales : leurs héritages se sont en effet largement diversifiés selon les lieux et les moments. Cette histoire est aujourd'hui en train de s'écrire; tous les articles de ce numéro montrent la richesse des sources manuscrites ou imprimées qui existent en ce domaine et qui, pour beaucoup, demeurent inédites, sans compter celles qui sont à découvrir dans des fonds d'archives ou des bibliothèques de toute l'Europe. Les lieux de dépôts de ces types de sources sont en effet particulièrement incertains.

L'article de Jean-Pierre Peter sur le marquis de Puységur ouvre le numéro parce que la découverte du somnambulisme magnétique en 1784 est directement issue du magnétisme animal. De la mise au jour de ce phénomène (pour lequel le médecin anglais James Braid crée le nom d'hypnose en 1843) vont naître de manière lente et tortueuse à la fois le concept freudien d'inconscient et toutes les formes de psychothérapie de la fin du XIX ${ }^{\mathrm{e}}$ siècle. Jean-Pierre Peter analyse ici les plis et les replis de cette découverte inattendue et de son déploiement qu'il éclaire de manière subtile, érudite et dérangeante tout en montrant l'ampleur de ses enjeux épistémologiques et philosophiques. Christine Bergé découvre ensuite le versant religieux, voire mystique, du somnambulisme magnétique lorsqu'à la fin du XVIII et au début du $\mathrm{XIX}^{\mathrm{e}}$ siècle, des francs-maçons se passionnent pour le magnétisme animal.

7. Ni les états allemands (et en particulier la Prusse), ni la Russie, ni le Danemark, ni l'Ecosse, ni plus tard l'Angleterre (voir Alison Winter, Mesmerized. Power of Mind in Victorian Britain, Chicago and London, The University of Chicago Press, 1998) ou les États-Unis ne mettent à l'écart le magnétisme de manière aussi péremptoire.

8. Voir Luis Montiel, Daemoniaca. Curacion magica, posesion y profecia en el marco de la magnetismo animal ramantico, Barcelone, MRA, 2006, en particulier p. 13-32. 
Elle propose ici une étude des nombreux cahiers manuscrits de la sœur de l'un d'entre eux, la chanoinesse Marie-Louise de Monspey (1733-1813) dite l'Agent inconnu. Ces écrits se veulent porteurs d'une "vraie» doctrine du magnétisme, livrant aussi une interprétation ésotérique de l'anatomie humaine et sont étudiés avec grande attention par des frères maçons des plus hauts grades. Les liens entre corps et âme, entre matière et esprit constituent l'un des questionnements récurrents révélés par le magnétisme aninal et ce dernier trouve ainsi dans l'Allemagne romantique du début du XIX ${ }^{\mathrm{e}}$ siècle, dans la génération des Naturphilosophen, un terrain propice à son développement. Tel est l'objet de l'article de Luis Montiel : comme dans celui de Jean-Pierre Peter, il est à nouveau question de la découverte de l'inconscient sous la forme d'une "conscience de l'involontaire». Il est à nouveau question également de la place des femmes, si particulière dans leurs liens avec le somnambulisme magnétique. Les somnambules sont en effet majoritairement des femmes, soit guérisseuses voyant les maux à l'intérieur des corps, soit patientes soignées par magnétisme. Les sources connues le montrent abondamment. Deux articles nous présentent plus en détail les maladies de deux femmes aux soins desquelles deux médecins ont participé de près ou de loin : Alexandre Bertrand (1795-1831), polytechnicien, médecin hospitalier à Paris et Antoine Despine (1777-1852), médecin-directeur des eaux d'Aixles-Bains. Jacqueline Carroy, en analysant la correspondance encore inédite d'une riche cliente suisse, Joséphine Lüdert, avec Antoine Despine nous permet de comprendre comment des médecins du début du XIX ${ }^{\mathrm{e}}$ siècle ont pu pratiquer et interpréter le magnétisme animal. Elle éclaire avec précision les types de soins donnés par Despine à ses patientes. C'est aussi à travers les notes et lettres de ce même médecin et de son confrère parisien Alexandre Bertrand que Jan Goldstein étudie la maladie de Nanette Leroux, patiente pauvre cette fois, puisqu'il s'agit d'une jeune campagnarde savoyarde soignée aux eaux d'Aix par charité. À travers ces deux études de cas, la complexité du magnétisme animal et de ses lectures par des historiennes contemporaines est bien mise en lumière. Enfin, dans un dernier article, je m'interroge sur les motifs de la mise à l'écart des faits magnétiques par les communautés académiques françaises. Au delà de raisons scientifiques, $j$ 'y analyse la réception du magnétisme animal en France et les possibles origines de sa condamnation, réitérée par deux fois, en 1784 et en 1837, en recherchant les facteurs culturels, sociaux et politiques de ce rejet, plus radical en France que dans d'autres espaces européens.

Ce numéro thématique, Savoirs occultés : du magnétisme à l'hypnose, propose donc une étude de la naissance du magnétisme animal à la fin du XVIII siècle et de son déploiement dans les premières décennies du XIX ${ }^{e}$ siècle. Il montre comment, autour des balbutiements de ces nouveaux savoirs médicaux, se constitue une culture appuyée sur des réseaux de sociabilité alliant médecins, patients, croyants ou curieux, et soutenue par des échan- 
ges constants et syncrétiques entre pratiques thérapeutiques, croyances et théorisations. On passe ainsi de l'électrisation aux bains, à la magnétisation ou à l'automagnétisation; on s'échange les somnambules lucides, on lit les livres de Swedenborg, de Fourier, de Saint-Simon, on fait des expériences fluidiques, on théorise... Tour à tour, sur des modes différents, les six articles de ce numéro nous guident dans la compréhension de ces phénomènes magnétiques mettant au jour des espaces sociaux et culturels, des débats, des questionnements, ou des rejets, qui, dans la seconde moitié du XIX eiècle, se diviseront plus nettement entre croyances et savoirs, donnant naissance à la religion spirite d'une part, et par le canal des recherches sur l'hystérie et l'hypnose d'autre part, à la découverte d'un inconscient psychique. Ce pourrait être le sujet d'un autre numéro thématique...

Bibliographie des premiers ouvrages contemporains publiés sur le magnétisme animal, par ordre chronologique :

Auguste Viatte, Les sources occultes du Romantisme. IIluminisme - Théosophie. 17701820, Paris, Librairie Honoré Champion, 1965-1969, 2 volumes.

Dominique Barrucand, Histoire de l'hypnose, Paris, Presses universitaires de France, 1967.

Alan Gauld, The Founders of Psychical Research, New York, Shocken Books, 1968.

Robert Darnton, Mesmerism and the End of the Enlighment in France, Cambridge (Mass.), Harvard University Press, 1968, traduction française La Fin des Lumières, Le mesmérisme et la révolution, Paris, Perrin, 1984.

Henri Frédéric Ellenberger, The Discovery of the Unconscious. The History and Evolution of Dynamic Pychiatry, New York, Basic Books, 1970, traduction française $\grave{A}$ la découverte de l'inconscient. Histoire de la psychiatrie dynamique, Villeurbanne, SIMEP, 1974, (nouvelle édition Paris, Fayard, 1994, sous le titre Histoire de la découverte de l'inconscient).

Léon Chertok et Raymond de Saussure, Naissance du psychanalyste. De Mesmer à Freud, Paris, Payot, 1973.

François Azouvi, «Sens et fonction épistémologique de la critique du magnétisme animal par les Académies", Revue d'histoire des sciences, 1976; édition, introduction et notes de Charles de Villers, Le magnétiseur amoureux, Paris, Vrin, 1978.

Franklin Rausky, Mesmer ou la Révolution thérapeutique, Paris, Payot, 1977.

Jean-Pierre Peter, «Un intermédiaire mal perçu : Franz Anton Mesmer (1734-1825), médecin trop guérisseur, guérisseur trop médecin", dans Les intermédiaires culturels. Actes du colloque du Centre méridional d'histoire sociale des mentalités et des cultures, 1978, Aix en Provence, Publications de l'université de Provence, 1981.

Adam Crabtree, Animal Magnetism, Early Hypnotism and Psychical Research, 17661825: an Annotated Bibliography, New York, Kraus International Publications, 1988.

Alan Gauld, A History of Hypnotism, Cambridge, Cambridge University Press, 1992 avec une bibliographie du magnétisme animal et de l'hypnotisme. 